\title{
What Defines High Quality Care for Patients with Chronic Hepatitis C and Why Should We Care?
}

\author{
Samuel B. Ho
}

Published online: 19 November 2013

(C) Springer Science+Business Media New York (Outside the USA) 2013

Chronic infection with the hepatitis $\mathrm{C}$ virus is prevalent in the United States, especially in the "baby boomer" generation (people born between 1945 and 1965), with $2.4 \%$ of the general population and $10.3 \%$ of patients under the care of the Veterans Administration (VA) chronically infected [1]. The number of patients with cirrhosis and advanced liver disease is expected to continue to increase in the United States, with a projected peak incidence in 2021 [2]. Mitigating this looming peak of disease burden are marked improvements in hepatitis $\mathrm{C}$ antiviral treatment, accompanied by a strikingly reduced incidence of liver-related complications and death [3, 4]. Unfortunately the adverse effects combined with the length and complexity of current antiviral treatments have greatly limited the number of patients that receive this life-saving treatment in VA and community clinics. Furthermore, the approach to treatment varies widely among practices. Thus, specific aspects of medical care as they ultimately translate into improved morbidity, mortality, or quality-of-life for patients with chronic hepatitis $\mathrm{C}$ need to be identified and addressed.

The study by Kanwal et al. [5] published in this issue of Digestive Diseases and Sciences provides a basis on which these questions can be addressed. The study correlates the results of two national surveys of VA practitioners conducted in 2006 and 2007 by the VA Hepatitis C Resource Center program, with patient and facility information derived from the VA HCV Clinical Case Registry (CCR) during 2003-2006. HCV care was assessed as 23 process measures encompassing pre-treatment evaluation,

S. B. Ho $(\varangle)$

Department of Medicine, VA San Diego Healthcare System, University of California, San Diego, San Diego, CA, USA

e-mail: Samuel.Ho2@va.gov; ho.samuel@sbcglobal.net preventive care, and antiviral treatment documented in CCR data. The main outcome variable was an indicator that a patient received $>50 \%$ of all indicated care. Multivariate analysis associated patient and facility factors with receipt of overall $\mathrm{HCV}$ care. On the basis of their analysis, the authors reported that patients cared for in facilities with $>8$ half-day clinics/week/1,000 patients were more likely to receive preventive care, antiviral treatment, and treatment monitoring; patients at a facility with $>3$ quality improvement initiatives received more preventive and comorbid care; and patients at facilities with gastroenterology-based HCV clinics were more likely to receive more pre-treatment care and antiviral treatment compared with other types of clinics. The latter finding most likely reflects the involvement of providers who are better informed or local "champions" for hepatitis C treatment. On the other hand, primary care and infectious disease-based clinics were associated with higher preventive and comorbid condition care. These associations remained significant for patients without comorbidities and also for patients with cirrhosis. These data supplement and build on a previous retrospective study by Kanwal et al. [6] of HCV registry records alone, which demonstrated that receipt of pretreatment process measures significantly correlates with higher treatment rates.

Processes and organizational factors measured in these studies reflect a greater engagement of patients and practitioners in the treatment process, but we do not know which factors are necessary and/or sufficient. Of the cited process and structural measures, which are the most important for increasing antiviral treatment or improved quality of life? How many process measures are needed? Does it matter if patients receive preventive and comorbid care if antiviral treatment rates do not change? What are the best methods and strategies for implementation of new 
practices in hepatitis clinics? The next logical step is to follow up these observations with prospective trials to identify the important components of care, the specific clinic protocols, and the optimal interventions needed to provide a solid improvement in clinical outcomes.

Since many patients do not have time to wait for all of these questions to be answered, solutions should also be sought now using existing knowledge and resources addressing all aspects of care. First, improvement of process measures such as preventive services and pre-treatment evaluation, clinic templates, clinical reminders, and automatic testing and referral protocols is often feasible and more easily accomplished [7]. Inexpensive patient selfmanagement classes improve components of $\mathrm{HCV}$ patient quality-of-life and readiness for treatment [8]. These quality measures are likely necessary to eventually improve outcomes, but are likely not sufficient in and of themselves. A more challenging finding of Kanwal et al. is that superior clinic resources are associated with more antiviral treatment and higher quality measures, since adequate staffing is needed to provide good care. Clinic leaders should consequently be aware of patient/staff and clinic availability, advocating for appropriate resources when needed. At my facility we have a single half-day clinic devoted to hepatitis $\mathrm{C}$ staffed by an interdisciplinary team, the backbone of which are four internists who devote one-half day to the clinic and work with nurse practitioners, a hepatologist, pharmacist, and one psychiatrist. Interdisciplinary clinics and collaborative models, such as the "patient centered medical homes" are effective for improving clinical processes for primary care and other complex diseases, such as diabetes, substance use, or HIV infection [9, 10]. An "integrated" care approach that provides mid-level case management in hepatitis $\mathrm{C}$ clinics may improve readiness for starting antiviral treatment $[11$, 12]. Antiviral treatment rates can be significantly increased in patients with psychiatric and substance use comorbidities if an integrated care protocol is used [13], an approach which should be considered by clinics interested in increasing treatment rates. More challenges exist in fee-forservice and resource-limited systems that lack the personnel, time, and money for examining their processes of care and implementing quality improvement interventions. The coming major shifts in the incentivization of health care delivery will likely improve the coordination of care and quality metrics that would ultimately help reduce the morbidity and mortality of hepatitis $\mathrm{C}$ patients.

The data presented by Kanwal et al. provide a highly detailed starting point to address quality improvement for hepatitis $\mathrm{C}$ patients. The ultimate effects of current health care reforms are difficult to predict. Nevertheless, everyone involved in the care of patients with hepatitis $\mathrm{C}$ should initiate quality improvement efforts in their own clinics, starting at all feasible levels, and keep focused on the goals of providing potentially life-saving treatment to as many patients as possible and for improving the quality-of-life for those unable to receive antiviral therapy.

\section{References}

1. Backus LI, Belperio PS, Loomis TP, et al. Hepatitis C Virus screening and prevalence among US veterans in Department of Veterans Affairs Care. JAMA Int Med. 2013;173:1549-1552.

2. Davis GL, Alter MJ, El-Serag H, et al. Aging of hepatitis C virus (HCV)-infected persons in the United States: a multiple cohort model of HCV prevalence and disease progression. Gastroenterology. 2010;138:513-521.

3. Backus LI, Boothroyd DB, Phillips BR, et al. A sustained virologic response reduces risk of all-cause mortality in patients with hepatitis C. Clin Gastroenterol Hepatol. 2011;9:509e1-516e1.

4. Morgan TR, Ghany MG, Kim HY, et al. Outcome of sustained virological responders with histologically advanced chronic hepatitis C. Hepatology. 2010;52:833-844.

5. Kanwal F, Hoang T, Chrusciel T, et al. Association between facility characteristics and the process of care delivered to patients with hepatitis C virus infection. Dig Dis Sci. (Epub ahead of print). doi:10.1007/s10620-013-2773-z.

6. Kanwal F, Hoang T, Chrusciel T, et al. Process of care for hepatitis $\mathrm{C}$ infection is linked to treatment outcome and virologic response. Clin Gastroenterol Hepatol. 2012;10:1270e3-1277e3.

7. Hagedorn H, Dieperink E, Dingmann D, et al. Integrating hepatitis prevention services into a substance use disorder clinic. $J$ Subst Abuse Treat. 2007;32:391-398.

8. Groessl EJ, Weingart KR, Stepnowsky CJ, et al. The hepatitis C self-management programme: a randomized controlled trial. $J$ Viral Hepat. 2012;18:358-368.

9. Ouwens M, Wollersheim H, Hermens R, et al. Integrated care programmes for chronically ill patients: a review of systematic reviews. Int J Qual Health Care. 2005;17:141-146.

10. DeVries A, Li CH, Sridhar G, et al. Impact of medical homes on quality, healthcare utilization, and costs. Am J Manag Care. 2012;18:534-544.

11. Knott A, Dieperink E, Willenbring ML, et al. Integrated psychiatric/medical care in a chronic hepatitis $\mathrm{C}$ clinic: effect on antiviral treatment evaluation and outcomes. Am J Gastroenterol. 2006;101:2254-2262.

12. Evon DM, Simpson K, Kixmiller S, et al. A randomized controlled trial of an integrated care intervention to increase eligibility for chronic hepatitis C treatment. Am J Gastroenterol. 2011;106:1777-1786.

13. Ho SB, Groessl EJ, Brau N, et al. Multisite randomized trial of an integrated care model for HCV patients with psychiatric and substance use co-morbidities: final results of impact on treatment initiation. Hepatology. 2012;56:1000A. 